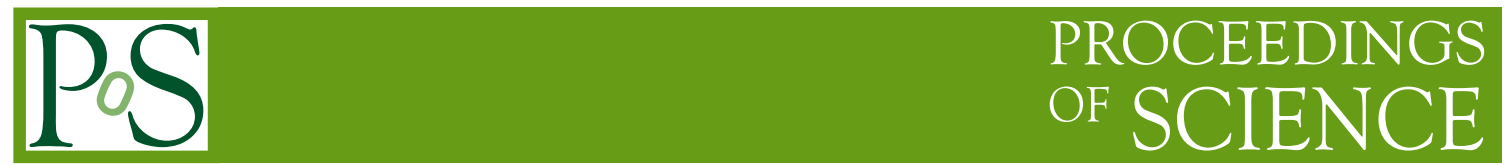

\title{
Higgs boson couplings and properties with CMS
}

\section{Linda Finco - on behalf of the CMS Collaboration*}

INFN and University of Torino, Via P. Giuria 1 Torino, Italy

E-mail: linda.finco@cern.ch

\begin{abstract}
Many different production and decay modes of the $126 \mathrm{GeV}$ mass Higgs boson have been studied by the CMS collaboration at the LHC collider. The analysis is based on pp collision data collected at center-of-mass energies of 7 and $8 \mathrm{TeV}$ corresponding to integrated luminosities of 5/fb and 20/fb respectively. The measurement of the Higgs boson couplings and of the study of its properties are presented.
\end{abstract}

XXII. International Workshop on Deep-Inelastic Scattering and Related Subjects, 28 April - 2 May 2014

Warsaw, Poland

${ }^{*}$ Speaker. 

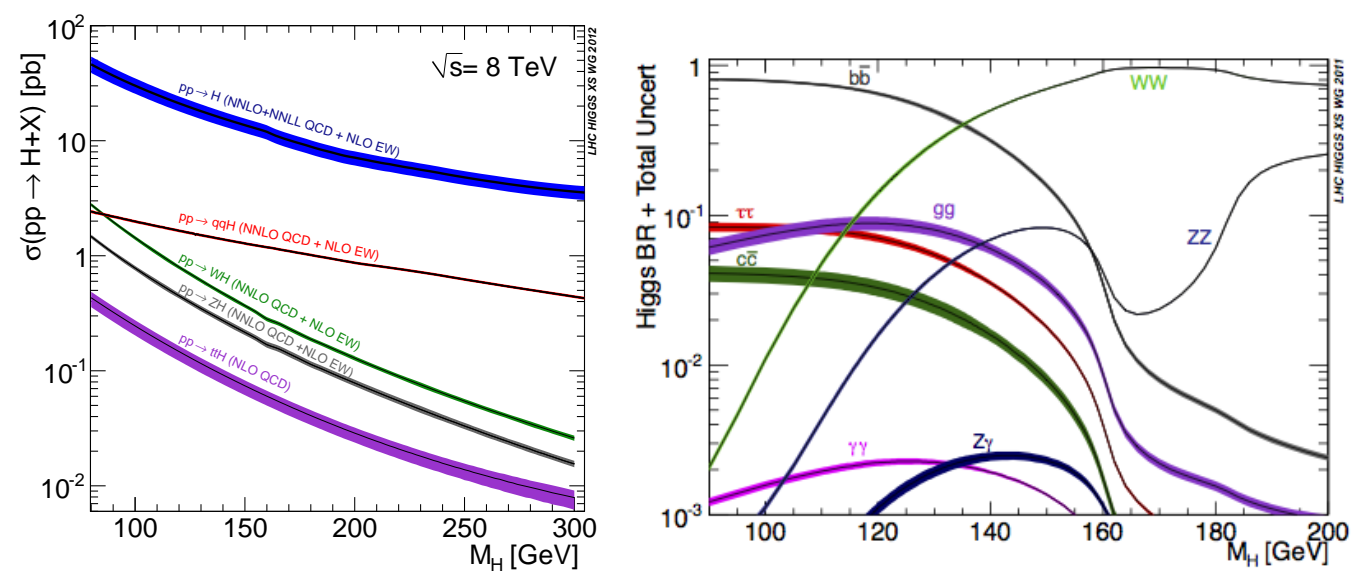

Figure 1: Standard Model Higgs boson production cross sections at $\sqrt{s}=8 \mathrm{TeV}$ (left). Standard Model Higgs boson decay branching ratios (right).

This article summarizes the Higgs boson [1] properties and couplings, measured by the Compact Muon Solenoid Experiment (CMS). The proton-proton collision data used in this analysis were recorded by the CMS detector at the Large Hadron Collider (LHC) and correspond to integrated luminosities of $5.1 f^{-1}$ at $\sqrt{s}=7 \mathrm{TeV}$ and $19.6 f^{-1}$ at $\sqrt{s}=8 \mathrm{TeV}$. After a brief overview on the production and decay modes, the Higgs boson properties (i.e. mass, spin-parity quantum numbers and width) are examined and the Higgs boson couplings with the other particles are analyzed, testing the validity of the Standard Model (SM) [2].

There are four main Higgs boson production modes in pp collisions at $\sqrt{s}=7-8 \mathrm{TeV}$ : the gluon-gluon fusion (which has the largest cross section), followed in turn by the vector boson fusion, the associated production with a $\mathrm{W}$ or $\mathrm{Z}$ boson and with a top quark pair (Fig 1, left). In the low mass range, five main decay channels are exploited: $H \rightarrow \gamma \gamma, H \rightarrow Z Z \rightarrow 4 \ell, H \rightarrow W W \rightarrow$ $\ell v \ell v, H \rightarrow \tau \tau$ and $H \rightarrow b \bar{b}$ (Fig 1, right).

\section{The Mass}

The mass is the most important property of the examined particle: its value is not determined by the SM, but once it is known, all the other characteristics are precisely predicted by the theory. The channels with the highest sensitivity for discovering the SM Higgs boson with the mass near to $125 \mathrm{GeV}$ are the $H \rightarrow \gamma \gamma$ and $H \rightarrow Z Z \rightarrow 4 \ell$ decay channels, thanks to their excellent mass resolution. The former is characterized by a modest branching fraction, a clear signature with two isolated and energetic leptons and a large background from QCD. The $H \rightarrow \gamma \gamma$ analysis [3] searches for a localized excess of diphoton events over a smoothly falling background, due to prompt diphoton production and to events with at least one jet misidentified as photon. The search is performed using MVA techniques, both for photon identification and event classification, and the signal is extracted from the background using a fit to the diphoton mass spectrum.

The $H \rightarrow Z Z \rightarrow 4 \ell$ channel [4] is characterized by a very small branching fraction and a very clean signature, represented by two pairs of high $p_{T}$ and isolated leptons, and its products are 

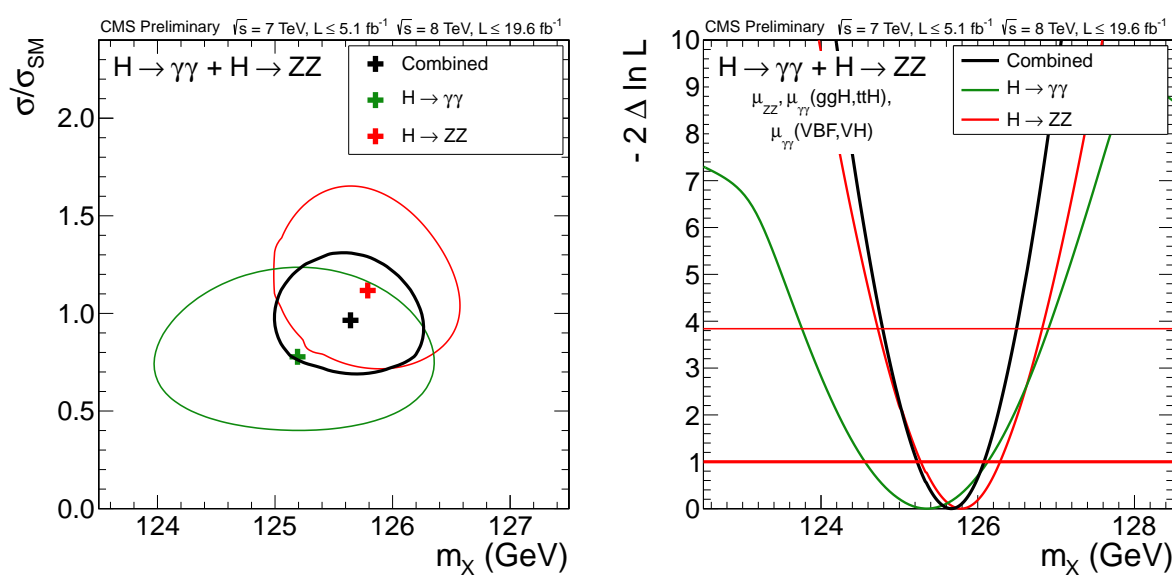

Figure 2: 2D 68\% CL contours for a hypothesized Higgs boson mass $m_{X}$ and signal strength $\sigma / \sigma_{S M}$ for the $\gamma \gamma$ and $4 \ell$ states separately and for their combination (left). 1D test statistics scan versus hypothesized Higgs boson mass $m_{X}$ for the $\gamma \gamma$ and $4 \ell$ states separately and for their combination (right).

all visible in the detector, allowing a fine reconstruction of the event topology, thanks also to the small background contribution. Events are categorized according to the lepton flavor and the mass measurement is performed using a 3D fit with the mass of the four-lepton system, the uncertainty estimated on a per-event basis and a kinematic discriminant that depends on the kinematic information from the production and decay.

Measurements obtained in the diphoton and four-lepton channels can be combined (Fig 2), leading to a value of $m_{H}=125.7 \pm 0.3$ (stat.) \pm 0.3 (syst.) GeV.

\section{The Spin and Parity}

The measurement of spin and parity quantum numbers is a very good test of SM compatibility, since theoretic predictions are very clear and foresee a $0^{+}$boson. Alternative hypotheses can be excluded using test statistics and the exploited channels for this measurement are $H \rightarrow Z Z \rightarrow 4 \ell$ and $H \rightarrow W W \rightarrow \ell v \ell v$. The former is the most sensitive channel. This analysis uses a 2D fit, depending on two kinematic discriminants based on angular information: one separates the SM Higgs boson from the background, while the other distinguishes between two alternative hypotheses. Twelve models are tested and the summary of the expected and observed values for the test statistic distributions is represented in Fig 3.

The characteristics of $H \rightarrow W W \rightarrow \ell v \ell v$ decay mode [5] are the distinct signature represented by two high $p_{T}$ and isolated leptons with a small opening angle and missing transverse energy due to undetected neutrinos, a very poor mass resolution and a large background. In the spin-parity analysis, categories with different lepton flavor and 0 or 1 jet in the event are used to distinguish between the SM and the $2^{+}$boson (produced in both $g g$ and $q \bar{q}$ processes) or the pseudoscalar hypothesis (produced in $g g$ fusion only). Two discriminant variables are used (transverse mass and dilepton mass) to separate spin hypotheses. In Fig. 4, distributions of the test statistic for the $0^{+}$and $2^{+}$ hypothesis are reported, shown for the case in which the $2^{+}$particle is produced via $g g$ fusion (left) 


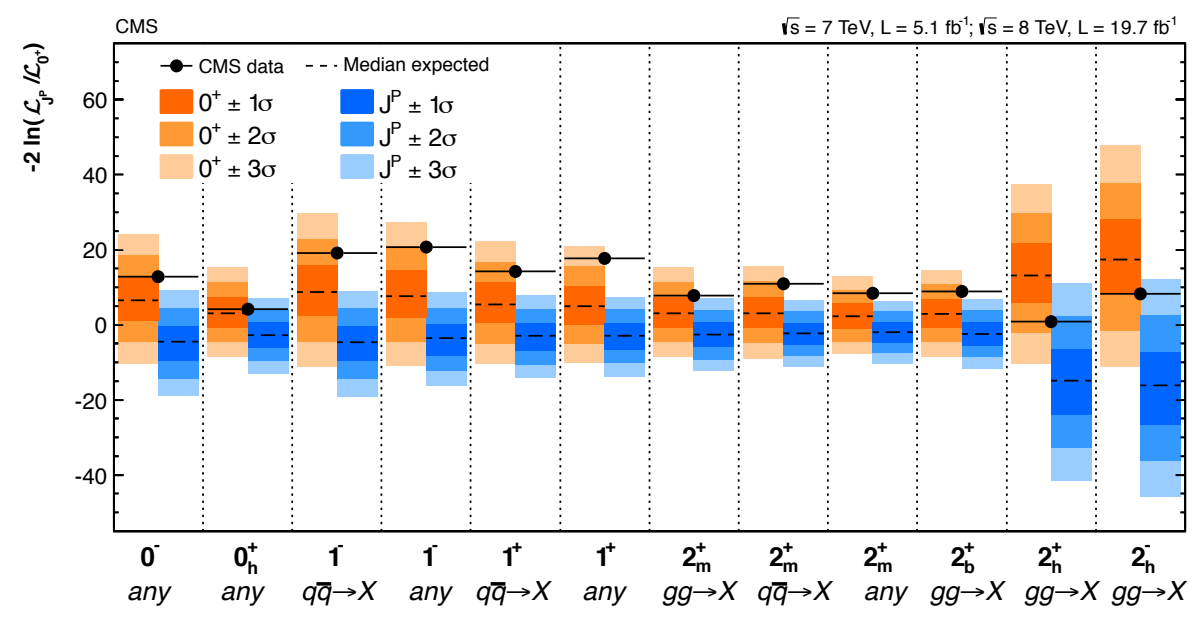

Figure 3: Summary of the expected and observed values for the test-statistic distributions for the twelve alternative hypotheses tested with respect to the SM Higgs boson, obtained using the $H \rightarrow Z Z \rightarrow 4 \ell$ analysis.
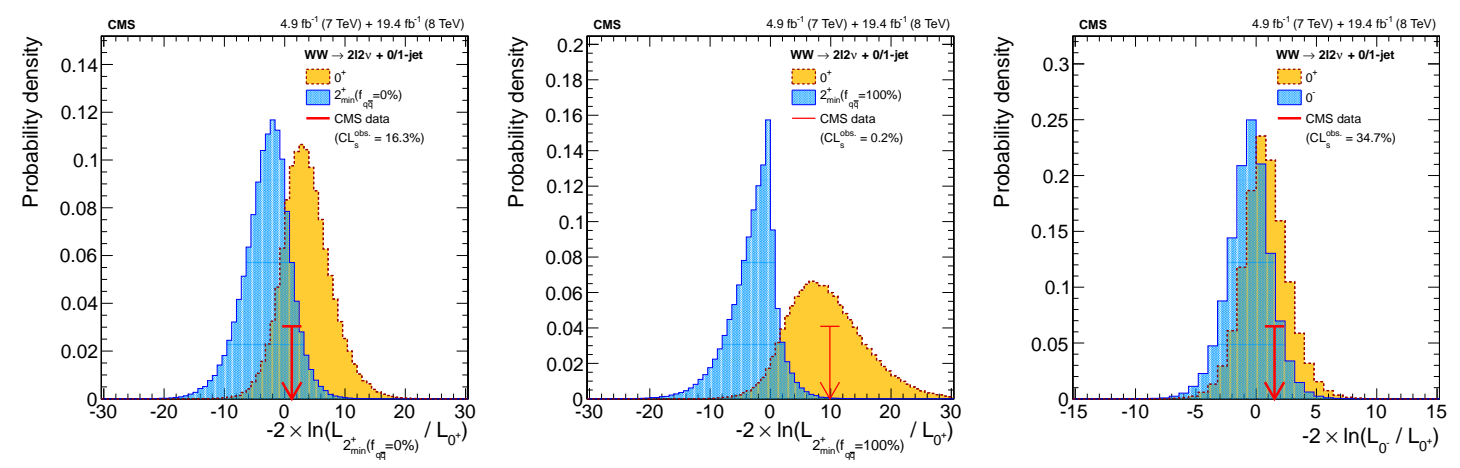

Figure 4: Distributions of the test statistic for the $0^{+}$vs $2^{+}$case, produced via $g g$ fusion (left) or $q \bar{q}$ annihilation (center) and distribution of the test statistic for the $0^{+}$vs $0^{-}$case (right), obtained using the $H \rightarrow W W \rightarrow \ell v \ell v$ analysis.

or $q \bar{q}$ annihilation (center), while in Fig. 4 (right) the same distributions are reported for the $0^{+}$ versus $0^{-}$case. The red arrow represents the observed value, that favors the SM hypothesis.

\section{The Width}

The Higgs width measurement is the most recent result of the CMS collaboration on the Higgs properties [6]. At $125.6 \mathrm{GeV}$ the $\mathrm{SM}$ predicts a width of about $4 \mathrm{MeV}$ and therefore a direct measurement is strongly limited by the experimental resolution. Indeed, up to now, constraints of 3.4 (6.9) $\mathrm{GeV}$ in the $H \rightarrow Z Z \rightarrow 4 \ell(H \rightarrow \gamma \gamma)$ channel have been reported. The idea is thus to measure the Higgs boson width using the Higgs boson production and decay away from the resonance, in the $4 \ell$ and $2 \ell 2 v$ final states. From the theory one can see that the resonant cross section depends on the signal strength $\mu$, while the off-shell cross section depends on the product of $\mu$ and $r$, the ratio of the observed width and the width predicted by the SM. Once the signal 


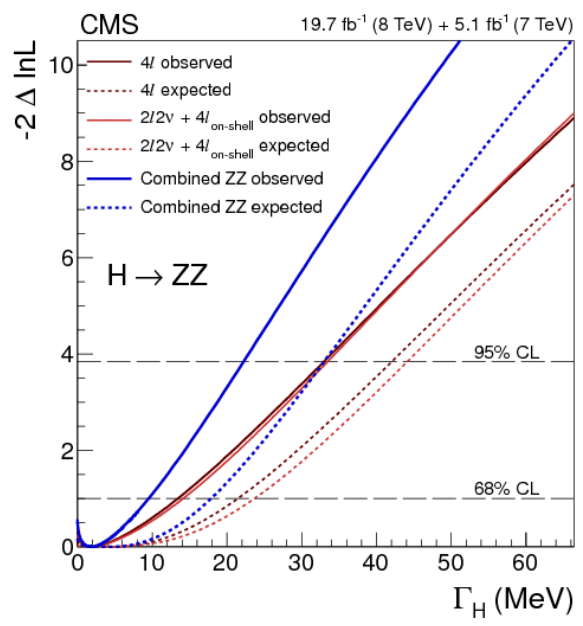

Figure 5: Scan of the negative log-likelihood, as a function of $\Gamma_{H}$ for the combined fit of the $4 \ell$ and $2 \ell 2 \mathrm{~V}$ channels (blue thick lines), for the $4 \ell$ channel alone in the off-shell and on-shell regions (dark red lines), and for the $2 \ell 2 v$ channel in the off-shell region and $4 \ell$ channel in the on-shell region (light red lines). The solid lines represent the observed values, the dotted lines the expected values.

strength is fixed using the value obtained from the data, $r$ value can be extracted by the ratio of the two cross sections, paying attention to the destructive interference with the continuum $g g \rightarrow Z Z$, which is not negligible at high masses. A likelihood is defined according to the final state and upper limits are obtained. In Fig. 5 the scan of the likelihood as a function of $\Gamma_{H}$ for the $4 \ell$ and $2 \ell 2 v$ final states is reported and the combination of the two channels leads to an observed constraint of 4.2 times the SM, corresponding to $\Gamma_{H}<17.4 \mathrm{MeV}$ at $95 \% \mathrm{CL}$.

\section{The Couplings}

The event yield in any production times decay mode is assumed to be related to the production cross section $\left(\sigma_{i i}\right)$ and the partial $\left(\Gamma_{f f}\right)$ and total $\left(\Gamma_{t o t}\right)$ Higgs boson decay width through the following equation

$$
\sigma \times B R(i i \rightarrow H \rightarrow f f)=\frac{\sigma_{i i} \Gamma_{f f}}{\Gamma_{t o t}} .
$$

The production cross section and the partial width are proportional to the square of the effective Higgs couplings to the corresponding particles. To test for possible deviations in the data from the rates expected in the different channels, modified couplings are introduced, denoted by scale factors $k_{i}$, and the data are fitted to these parameters [2]. Significant deviations of any $k_{i}$ from unity would imply new physics. One can thus compare the observation with the expectation, by fitting for two parameters $\left(k_{V}\right.$ and $\left.k_{f}\right)$, modifiers for all Higgs boson couplings to vector bosons and fermions. At leading order all partial widths, except for $\Gamma_{\gamma \gamma}$, scale either as $k_{V}^{2}$ or $k_{f}^{2}$. On the other hand, $\Gamma_{\gamma \gamma}$ is induced through $\mathrm{W}$ and top loop diagrams and it scales as $\left|\alpha k_{V}+\beta k_{f}\right|^{2}: H \rightarrow \gamma \gamma$ is thus the only channel sensitive to the relative sign of $k_{V}$ and $k_{f}$. In Fig. 6 (left), the $68 \%$ CL contours for individual channels and for the overall combination is shown for the $\left(k_{V}, k_{f}\right)$ parameters.

Processes induced by loop diagrams can be particularly susceptible to the presence of new physics, 

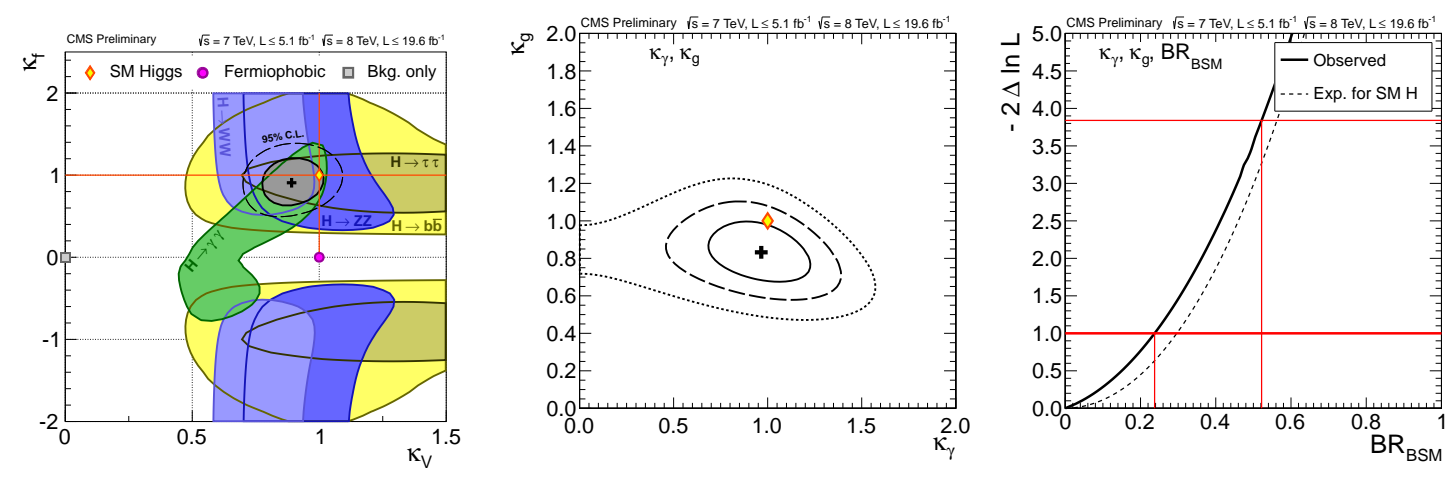

Figure 6: $68 \% \mathrm{CL}$ contours for individual channels (colored swaths) and for the overall combination (solid line) for the $\left(k_{V}, k_{f}\right)$ parameters. The cross indicates the global best-fit value and the yellow diamond shows the SM expectation (left). 2D likelihood scan for the $k_{g}$ and $k_{\gamma}$ parameters (center). The solid, dashed and dotted contours show the $68 \%, 95 \%$ and $99.7 \%$ CL regions, respectively. Likelihood scan versus $B R_{B S M}=\Gamma_{B S M} / \Gamma_{t o t}$ (right).

thus data can be combined and fitted for the scale factors $k_{\gamma}$ and $k_{g}$. Results are reported in Fig 6 (center) and they are compatible with the SM prediction. Moreover, the Higgs boson could also decay into invisible particles or particles not detectable at the LHC. A modified total Higgs boson width is thus defined, containing also the branching ratio of not-detectable processes, and left free to float in the fit. Results are shown in Fig 6 (right) and they are compatible with the SM.

In summary, the mass of the analyzed resonance is measured with high precision. The particle is compatible within uncertainties with a SM Higgs boson and alternative spin-parity hypotheses are disfavored by the data. The experimental constraint on Higgs total width is determined using off-shell production and decay, improving by more than two orders of magnitude the previous experimental result. A comprehensive set of Higgs coupling fits is reported and no significant deviation from SM predictions is observed within the uncertainties.

\section{References}

[1] The CMS Collaboration, Observation of a new boson at a mass of $125 \mathrm{GeV}$ with the CMS experiment at the LHC, Phys. Lett. B 716 (2012) 30, arXiv:1207.7235

[2] The CMS Collaboration, Measurements of the properties of the new boson with a mass near $125 \mathrm{GeV}$, CMS-PAS-HIG-13-005

[3] The CMS Collaboration, Properties of the observed Higgs-like resonance decaying into two photons, CMS-PAS-HIG-13-016

[4] The CMS Collaboration, Measurement of the properties of a Higgs boson in the four-lepton final state, arXiv: 1312.5353

[5] The CMS Collaboration, Measurement of Higgs boson production and properties in the WW decay channel with leptonic final states, JHEP 01 (2014) 096, arXiv:1312.1129

[6] The CMS Collaboration, Constraints on the Higgs boson width from off-shell production and decay to

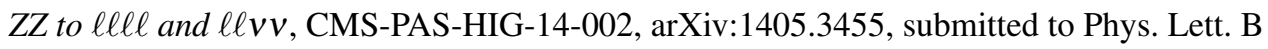

